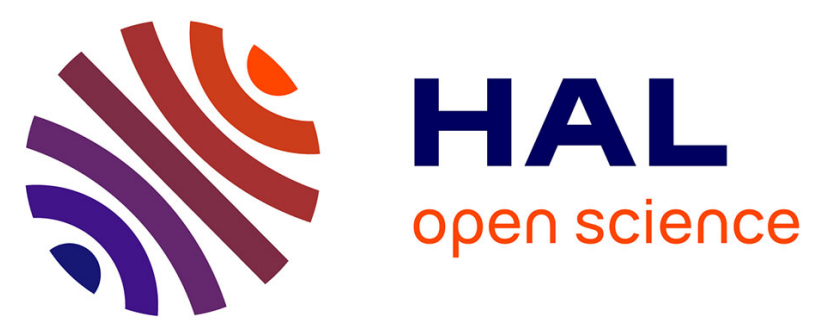

\title{
Novel FH mutations in families with hereditary leiomyomatosis renal cell cancer (HLRCC) and in patients with isolated type 2 papillary renal cell carcinoma
}

Betty Gardie, Audrey Remenieras, Darouna Kattygnarath, Johny Bombled, Sandrine Lefèvre, Victoria Perrier-Trudova, Pierre Rustin, Michel Barrois, Abdelhamid Slama, Marie-Francoise Avril, et al.

\section{- To cite this version:}

Betty Gardie, Audrey Remenieras, Darouna Kattygnarath, Johny Bombled, Sandrine Lefèvre, et al.. Novel FH mutations in families with hereditary leiomyomatosis renal cell cancer (HLRCC) and in patients with isolated type 2 papillary renal cell carcinoma. Journal of Medical Genetics, 2011, 48 (4), pp.226. 10.1136/jmg.2010.085068 . hal-00614499

\author{
HAL Id: hal-00614499 \\ https://hal.science/hal-00614499
}

Submitted on 12 Aug 2011

HAL is a multi-disciplinary open access archive for the deposit and dissemination of scientific research documents, whether they are published or not. The documents may come from teaching and research institutions in France or abroad, or from public or private research centers.
L'archive ouverte pluridisciplinaire HAL, est destinée au dépôt et à la diffusion de documents scientifiques de niveau recherche, publiés ou non, émanant des établissements d'enseignement et de recherche français ou étrangers, des laboratoires publics ou privés. 


\section{Novel $F H$ mutations in families with hereditary leiomyomatosis and renal cell cancer}

(HLRCC) and patients with isolated type 2 papillary renal cell carcinoma

Corresponding author: Prof. Stéphane Richard, Génétique Oncologique EPHE, Faculté de

Médecine Paris-Sud, 63 avenue du Général Leclerc, Le Kremlin-Bicêtre, France; stephane.richard@u-psud.fr Phone: 331495967 28, Fax: 33149596726.

Betty Gardie 1 , Audrey Remenieras ${ }^{2}$, Darouna Kattygnarath ${ }^{2}$, Johny Bombled ${ }^{2}$, Sandrine Lefèvre ${ }^{1}$, Victoria Perrier-Trudova ${ }^{1}$, Pierre Rustin ${ }^{3}$, Michel Barrois ${ }^{2}$, Abdelhamid Slama ${ }^{4}$, Marie-Françoise Avril 5,41 , Didier Bessis 6 , Olivier Caron ${ }^{7}$, Frédéric Caux 8 , Patrick Collignon $^{9}$, Isabelle Coupier 10,41, Carol Cremin 11 , Hélène Dollfus 12,41 , Catherine Dugast $^{13}$, Bernard Escudier 14,41, Laurence Faivre ${ }^{15}$, Michel Field 16 , Brigitte GilbertDussardier 17,41, Nicolas Janin 18, Yves Leport ${ }^{19}$, Dominique Leroux ${ }^{20}$, Dan Lipsker ${ }^{21}$, Félicia Malthieu ${ }^{22}$, Barbara McGilliwray ${ }^{11}$, Christine Maugard 23 , Arnaud Méjean24,41, Isabelle Mortemousque 25, Ghislaine Plessis 26, Bruce Poppe 27, Christelle PruvostBalland ${ }^{28}$, Serena Rooker ${ }^{29}$, Joelle Roume ${ }^{30}$, Nadem Soufir ${ }^{31}$, Michelle Steinraths ${ }^{32}$, Min-Han Tan ${ }^{33}$, Christine Théodore ${ }^{34}$, Luc Thomas ${ }^{35}$, Pierre Vabres ${ }^{36}$, Emmanuel Van Glabeke 37 , Jean-Baptiste Meric 38 , Virginie Verkarre 39,42 , Gilbert Lenoir ${ }^{2}$, Virginie Joulin 40, Sophie Deveaux ${ }^{42}$, Veronica Cusin 2,42, Jean Feunteun 40 , Bin Tean Teh ${ }^{41}$, Brigitte Bressac-de Paillerets 2 , , Stéphane Richard $1,42 *$ on behalf of the French National Cancer Institute "Inherited predisposition to kidney cancer" network.

1 - Génétique Oncologique EPHE, INSERM U753, Institut de cancérologie Gustave Roussy Villejuif ; and Faculté de Médecine Paris-Sud, Le Kremlin-Bicêtre, France

2 - Service de Génétique, Institut de cancérologie Gustave Roussy, Villejuif, France

3 - INSERM, U676, Paris, and Université Paris 7, Faculté de médecine Denis Diderot, IFR02, Paris, France

4 -Laboratoire de Biochimie, Hôpital de Bicêtre, AP-HP, Le Kremlin-Bicêtre, France

5 - Service de Dermatologie, Hôpital Cochin, AP-HP, Paris, France

6 - Service de Dermatologie, Hôpital Saint-Eloi, Montpellier, France

7 - Consultation d'Oncogénétique, Institut de cancérologie Gustave Roussy, Villejuif, France

8 - Service de Dermatologie, Hôpital Avicenne, AP-HP, Bobigny, France

9 - Service de Génétique Médicale, Centre Hospitalier Intercommunal, Toulon, France

10 - Service d'Oncogénétique et Génétique Médicale, Hôpital A. de Villeneuve, Montpellier, France

11 - BC Cancer Agency, Vancouver, Canada

12 - Service de Génétique Médicale, Hôpital Civil, Strasbourg, France 
13 - Consultation d'Oncogénétique, CHU, Rennes, France

14 - Département d'Immunothérapie, Institut de cancérologie Gustave Roussy, Villejuif, France

15 - Centre de Génétique, CHU Hôpital d'Enfants, Dijon, France

16 - Genetic Services, Royal North Shore Hospital, St Leonards, Australia

17 - Service de Génétique, CHU, Poitiers, France

18 - Département de Génétique, CHU, Liège, Belgique

19 - Cabinet de Dermatologie, Vire, France

20 - Service de Génétique Médicale, CHU Michalon, Grenoble, France

21 - Service de Dermatologie, Hôpital Civil, Strasbourg, France

22 - Centre médico-chirurgical Atlantique, Puilboreau, France

23 - Laboratoire de diagnostic génétique, Hôpital Universitaire de Strasbourg, Strasbourg, France ; and CR-CHUM, Université de Montréal, Montréal (QC), Canada

24 - Service d'Urologie, Hôpital Necker, AP-HP, Paris, France

25 - Service de Génétique Médicale, Hôpital Bretonneau, Tours, France

26 - Service de Génétique, CHU Clémenceau, Caen, France

27 - Center for Medical Genetics, Universiteit Gent, Gand, Belgique

28 - Service de Dermatologie, Hôpital Saint Louis, AP-HP, Paris, France

29 - Molecular Genetics Laboratory, Capital Coasts DHB, Wellington South, New Zealand

30 - Consultation de Génétique Médicale, Hôpital Ambroise Paré, AP-HP, Boulogne, France

31 - Consultation de Génétique, Hôpital Bichat-Claude Bernard, AP-HP, Paris, France

32 - Division of Medical Genetics, Victoria General Hospital, Victoria, BC, Canada

33 - Department of Medical Oncology, National Cancer Centre, Singapore

34 - Service d'Oncologie Médicale, Hôpital Foch, Suresnes, France

35 - Service de Dermatologie, Centre Hospitalier Sud, Hospices Civils, Lyon, France

36 - Service de Dermatologie, Hôpital du Bocage, CHU, Dijon, France

37 - Service d'Urologie, Hôpital André Grégoire, Montreuil, France

38 - Service d'Onco-Hématologie, Centre Médical de Bligny, Briis sous Forges, France

39 - Laboratoire d'Anatomie Pathologique, Hôpital Necker-Enfants Malades, AP-HP,

Paris, France

40 - Stabilité génétique et oncogénèse, CNRS UMR 8200, Institut de cancérologie

Gustave Roussy, Villejuif, France

41 - Van Andel Research Institute, Grand Rapids, Michigan, USA

42 - Centre Expert National Cancer Rares "PREDIR", Institut National du Cancer (INCa),

Service d'Urologie, Hôpital de Bicêtre, AP-HP, Le Kremlin- Bicêtre, France

KEY WORDS: leiomyomatosis, $F H$, HLRCC, papillary renal cell cancer type II

WORDS COUNT: 3435 


\section{ABSTRACT: 242}

\section{Background}

Hereditary leiomyomatosis and renal cell cancer (HLRCC) is an autosomal dominant disorder predisposing humans to cutaneous and uterine leiomyomas; in $20 \%$ of affected families, type 2 papillary renal cell cancers (PRCCII) also occur with aggressive course and poor prognosis. HLRCC results from heterozygous germline mutations in the tumor suppressor fumarate hydratase $(F H)$ gene.

\section{Methods}

As part of the French National Cancer Institute (INCa) "Inherited predispositions to kidney cancer" network, we performed sequence analysis and a functional study of $F H$ in 56 families with clinically proven or suspected HLRCC and in 23 patients with isolated PRCCII (5 familial and 18 sporadic).

\section{Results}

We identified 32 different germline $F H$ mutations (15 missense, six frameshifts, four nonsense, one deletion/insertion, five splice site and one complete deletion) in 40/56 (71.4\%) families with proven or suspected HLRCC and in 4/23 (17.4\%) probands with PRCCII alone, including 2 sporadic cases. Twenty-one of these were novel and all were demonstrated as deleterious by significant reduction of FH enzymatic activity. In addition, five asymptomatic parents in three families were confirmed as carrying disease-causing mutations.

\section{Conclusions}

This study identified and characterized 21 novel $F H$ mutations and demonstrated that PRCCII can be the only one manifestation of HLRCC. Due to the incomplete penetrance of HLRCC, we propose to extend the $F H$ mutation analysis to every patient with PRCCII 
occurring before $40 \mathrm{yrs}$ of age or when renal tumor harbors characteristic histologic features, in order to discover previously ignored HLRCC-affected families. 


\section{INTRODUCTION}

Hereditary leiomyomatosis and renal cell carcinoma (HLRCC, OMIM 605839) is an autosomal dominant familial disorder characterized by the development of cutaneous and uterine (fibroid) leiomyomas, renal cell carcinoma (RCC), and rarely uterine leiomyosarcomas.[1, 2] HLRCC was previously called multiple cutaneous and uterine leiomyomatosis (MCUL, OMIM 150800), as the association between skin and uterine leiomyomas was described before the discovery of RCC predisposition. Cutaneous leiomyomas occur in $76 \%$ of individuals at a mean age of 25 years (range: 10-47 years) but $40 \%$ percent of individuals have mild cutaneous manifestations with five or fewer lesions. $[3,4]$ Uterine leiomyomas are present in almost all women with a mean age at diagnosis of 30 yrs (range: $18-52$ years). [3-5] RCC occurrence is relatively low $(20 \%, 29 / 144$ families worldwide) and differs between and within families affected by HLRCC.[1, 3, 4, 6-24] RCCs predominantly affect young ( $<40$ years) adults with a mean age at diagnosis of 46 yrs (range: 17-75 years). [25] They are usually solitary, unilateral, and highly aggressive with rapid dissemination. The main histological RCC subtype is type 2 papillary RCC (PRCCII), a variety of renal cancer characterized by large tumor cells with eosinophilic cytoplasm and pseudostratified nuclei.[17, 25, 26] Less frequently, collecting duct RCC may also be observed in patients with HLRCC.[4, 25]

MCUL/HLRCC is associated with heterozygous germline mutations in the fumarate hydratase (or fumarase, $F H$ ) gene located at 1q42.3-q43.[2, 3, 6] Interestingly, homozygous and compound heterozygous $F H$ mutations have been first identified in fumarase deficiency, a rare autosomal recessive disorder characterized by neurological impairment and death in the first decade of life (FHD; MIM 136850, OMIM 606812). [27-29] FH spans $22 \mathrm{~kb}$, contains 10 exons, and encodes two fumarase isozymes, mitochondrial and cytosolic. The active form of $\mathrm{FH}$ is a homotetramer in which three of the four chains combine to form the enzymatic active site.[7, 30, 31] FH catalyzes the conversion of fumarate to malate in the mitochondrial matrix as part of the tri-carboxylic acid (TCA) 
cycle. Thus, FH deficiencies result in chronic accumulation of fumarate and altered levels of other TCA intermediates.

Fumarate accumulation has been shown to induce activation of hypoxia-inducible factor (HIF) and its target genes.[32] HIF plays a major role in the tissue response to hypoxia by inducing expression of multiple genes involved in cell survival and proliferation. In renal cancers and fibroids from HLRCC patients, alpha subunits of HIF (HIF1a and 2a) are overexpressed.[32, 33] Moreover, mice with inactive Fhl in the kidney developed proliferative renal cysts overexpressing HIF alpha subunits and hypoxia pathway factors.[34] FH alteration induces fumarate accumulation and the production of reactive oxygen species, leading to activation of the HIF alpha subunits through inhibition of prolyl hydroxylase (PHD2), triggering the VHL-dependent degradation of HIF alpha subunits under normoxia.[35] Therefore, $F H$ inactivation seems to be implicated in inappropriate activation of oncogenic hypoxia pathways, similar to the manner in which $V H L$ germline mutations result in von Hippel-Lindau disease, the main cause of hereditary clear cell RCC (CCRCC). However, in contrast to the frequent observation of somatic VHL mutations in sporadic CCRCCs, to date no FH mutations have been detected in sporadic PRCCIIs.[36]

To date, four complete deletions of $F H$, one exon deletion, one exon duplication, and 81 different $F H$ germline point mutations have been reported in 144 MCUL/HLRCC families (Supplementary Table S1).[1, 3, 4, 6-24] [37, 38] There is no obvious relationship between genotype and the aggressiveness of the disease. All these mutations lead to the loss of FH enzymatic activity; the remaining functional allele is lost in most cutaneous, uterine, and renal tumors arising in patients carrying $F H$ germline heterozygous mutations.[6, 7] The function of $F H$ is then consistent with the function of tumor suppressor genes.[6] 
As part of the French National Cancer Institute (INCa) "Inherited predispositions to kidney cancer" network, we performed the first comprehensive genetic and functional analysis of FH in a large series of patients with phenotypes highly suggestive of MCUL/HLRCC disease and in patients with only PRCCII. These analyses enhance our knowledge of the FH germline mutational spectrum and expand the group of phenotypic features associated with these $F H$ mutations.

\section{MATERIALS AND METHODS}

\section{Patient selection}

Families were selected for inclusion in the study through two clinical approaches. Dermatology departments identified and recruited 56 families with clinical histories demonstrative $(\mathrm{N}=44)$ or suggestive $(\mathrm{N}=12)$ of MCUL/HLRCC. We defined a family as clinically affected if at least one member had more than 10 skin lesions clinically compatible with leiomyomas including a minimum of 1 lesion histologically confirmed. Patients with single cutaneous leiomyoma, isolated or associated with personal or familial uterine leiomyomas or RCC were classified as suggestive of potential MCUL/HLRCC. All alive patients had a detailed examination of the skin. Uterine fibroids and renal tumours were documented by history and review of medical records. Renal ultrasound or MRI was performed in all adult patients. A transvaginal ultrasound was also performed in all women who still had a uterus. In addition, urology departments recruited 18 patients with apparent sporadic PRCCII and five patients with familial PRCCII.

All patients were monitored by French physicians, with the exception of six families who were followed by international colleagues (Singapore, Australia, Belgium, Canada, and 
New Zealand). This study was approved by the Ethical Committee of Le Kremlin-Bicêtre University Hospital, France. All patients had previously provided informed consent for genetic testing and use of their DNA for further investigation. Blood samples from 180

unaffected French Caucasian individuals were used as controls to estimate the frequency of SNP and missense variants not available from the HapMap Project. For probands carrying the $F H$ mutation, targeted sequencing of $F H$ was extended to relatives (58 additional patients).

\section{Genomic rearrangement screening}

DNA was extracted from peripheral blood leukocytes according to standard procedures using the QIAamp DNA Blood Midi Kit (Qiagen, Valencia, CA).Quantitative real-time PCR based on SYBR-Green fluorescence technology was used to detect genomic rearrangements (large deletions or duplications of one exon or more). PCR was performed with the QuantiFast SYBR Green PCR Kit (Qiagen) on an ABI 7700 Sequence Detection System (Applied Biosystems). Primers designed for sequencing analysis were used at a concentration of $300 \mathrm{nM}$. A total of $20 \mathrm{ng}$ DNA was used in a $25 \mu \mathrm{L}$ reaction volume. The BRCAl gene was used as an internal reference. The copy number was determined using the 2- $\triangle \mathrm{CT}$ method where $\triangle \mathrm{CT}=\mathrm{CT} F H$ amplicon - CTreference gene. [39] 


\section{Sequencing analysis}

Mutations in $F H$ gene were screened by genomic DNA amplification of each exon and splice junction (primer sequences and PCR conditions available upon request). PCR products from genomic rearrangement screening were first purified using the ExoSAP-IT PCR purification kit (USB) and then sequenced with the Big Dye Terminator v.3.1 kit (Applied Biosystems). Resin Sephadex G50 superfine (Amersham) was used for purification. Sequencing was performed on an ABI3730 automatic DNA sequencer (Applied Biosystems) in 96-well plates. Variants and mutations were identified by visual inspection of the sequence with Seqscape 2.5 software (Applied Biosystems).

\section{Measurement of FH enzyme activity}

Lymphoblastoid cell lines (LCL) were generated by Epstein-Barr virus transformation of leukocytes from 20 patients with novel missense or splice mutations and eight wild-type controls. Peripheral blood lymphocytes (PBL) were isolated on Ficoll cushion. LCL and PBL were homogenized in a lyses buffer $(50 \mathrm{Mm}$ Tris- $\mathrm{HCl} \mathrm{pH} 7.2$ containing $10 \%$ Triton X100, 2mM Phenylmethylsulfonyl fluoride and 0.02\% of 2-mercaptoethanol) and subjected to brief sonication. Samples were centrifuged at $10.000 \mathrm{~g}$ for $20 \mathrm{mn}$ at $4^{\circ} \mathrm{C}$ and the supernatants were used for the enzyme assay. FH enzymatic activity was measured spectrophotometrically according to standard procedures. [40, 41] Briefly the assay monitors the increase in absorbance at $250 \mathrm{~nm}$ due to fumarate production from malate, with a final reaction medium consisting of $50 \mathrm{mM}$ malate, $50 \mathrm{mM}$ phosphate buffer $\mathrm{pH} 7.8$, The FH activity was reported as the amount of fumarate generated per min per milligram of 
protein, or in a ratio to the corresponding citrate synthase activity, final results are then expressed as percentage of control activity.

\section{RESULTS}

\section{Mutation analysis}

FH genotyping was carried out on germline DNA and sequencing of the entire gene (coding sequence and exon/intron junctions) revealed 31 different sequence variations in 40/56 families (71.4\%) with clinically proven or suspected HLRCC and in 4/23 patients (17.4\%) with only PRCCII (2/18 sporadic and 2/5 familial) including the first Chinese origin family described (family F44). The identified mutations included 15 missense, six frameshifts, four nonsense, one deletion/insertion and five splice site mutations. Country of origin of families, molecular results and references are summarized in Table 1.

We completed the $F H$ mutational spectrum by exploring large deletion or duplication events and identified one patient with a complete $F H$ deletion (proband of family F1, Table 1 and Figure 1).

Twenty-one of the identified mutations have never been described previously (noted "this report" in Table 1), and none of these mutations was found in control DNA samples (0/360 alleles sequenced). These novel mutations included seven missense (p.Leu168Pro, p.His192Arg, p.Gln211Arg, p.Phe269Ser, p.Leu272Pro, p.Asn330Asp, p.Gln343Arg), two nonsense (p.Glu270X, p.Trp457X), six frameshifts (c.127_128delGA, c.47delT, c.298delA, c.666delC, c.810delA, c.994delA), one insertion-deletion (p.Leu374_His375insdelTyr) and five splice site (138+1_138+10del10, 247_249+1delGAGGinsA, 250-2A>G,426+1G>A, 
1108-2A>G). Another mutation (p.Ala274Val) was identified by our group during the study but was published as a case report. [42] Four novel mutations were found in unrelated French families (F9 and F10; F11, F12 and F13; F24 and F25; F32 and F33, respectively) from different areas and there was no evidence of common ancestors. Interestingly, 3 out of 4 mutations identified in probands with isolated PRCCII were also novel (Table 1).

We then performed an in silico analysis of the putative functional consequences of the missense mutations. As shown in Figure 2, all $F H$ missense mutations identified in the present study affect residues that have been highly conserved throughout evolution.

\section{Functional characterization of novel mutations}

In order to demonstrate the functional consequences of germline $F H$ mutations, we measured the enzymatic activity of endogenous $\mathrm{FH}$ in peripheral blood lymphocytes or lymphoblastoid cells derived from patients. We tested 30 patients carrying an $F H$ mutation

(including 15 unpublished mutations, of which eight are novel missense mutations) and eight samples of the wild-type $F H$ gene. Reduction of enzymatic activity by at least $50 \%$ was observed for all mutations tested (Table 1). We did not notice a major difference in the enzymatic activities of missense mutations (from $34.6 \%$ to $54.7 \%$ activity compared to a wild-type control, mean activity 45\%) and total loss-of-function mutations such as deletions, nonsense mutations, or splice site mutations (from $39.5 \%$ to $60.8 \%$, mean activity $50.1 \%)$. 


\begin{tabular}{|c|c|c|c|c|c|c|}
\hline Family & Origin & Nucleotide change & $\begin{array}{c}\text { Aminoacid change } \\
\text { (HGVS) }\end{array}$ & Location & $\begin{array}{c}\text { FH } \\
\text { activity } \\
(\%)\end{array}$ & $\begin{array}{c}\text { First } \\
\text { description }\end{array}$ \\
\hline \multicolumn{7}{|c|}{ Families with demonstrative manifestations of HLRCC } \\
\hline F1 & France & c.1-?_1404+?del & p.Met1-?_X468+?del & NA & 41.3 & {$[6,7,38]$} \\
\hline $\mathrm{F} 2$ & France & c.127_128delGA & p.Glu43fs & Exon 2 & $42 *$ & This report \\
\hline F3 & Portugal & c.138+1_138+10del10 & Splice site & Intron 2 & $50 *$ & This report \\
\hline F4 & France & c.147delT & p.Ile50fs & Exon 3 & 39.5 & This report \\
\hline F5 & Australia & c. $172 \mathrm{C}>\mathrm{T}$ & p.Arg58X & Exon 3 & NA & $\begin{array}{c}{[4,6,14} \\
24]\end{array}$ \\
\hline F6 & France & c. $220 \mathrm{G}>\mathrm{C}$ & p.Ala74Pro & Exon 3 & NA & [6] \\
\hline F7 & $\begin{array}{c}\text { New } \\
\text { Zealand }\end{array}$ & c. $220 \mathrm{G}>\mathrm{C}$ & p.Ala74Pro & Exon 3 & NA & {$[6]$} \\
\hline F8 & France & c. $220 \mathrm{G}>\mathrm{C}$ & p.Ala74Pro & Exon 3 & NA & [6] \\
\hline F9 & France & $\begin{array}{c}\text { c.247_249+1 } \\
\text { delGAGGinsA }\end{array}$ & Splice site & Exon 3 & NA & This report \\
\hline F10 & France & $\begin{array}{c}\text { c.247_249+1 } \\
\text { delGAGGinsA }\end{array}$ & Splice site & Exon 3 & 57.2 & This report \\
\hline F11 & France & c. $250-2 A>G$ & Splice site & Intron 3 & 49.2 & This report \\
\hline F12 & France & c. $250-2 \mathrm{~A}>\mathrm{G}$ & Splice site & Intron 3 & 42.8 & This report \\
\hline F13 & France & c. $250-2 A>G$ & Splice site & Intron 3 & NA & This report \\
\hline F14 & Cambodia & c.298delA & p.Thr100fs & Exon 4 & $45^{*}$ & This report \\
\hline F15 & France & c. $410 A>G$ & p.His137Arg & Exon 4 & $44 *$ & [6] \\
\hline F16 & Maroc & c. $503 \mathrm{~T}>\mathrm{C}$ & p.Leu168Pro & Exon 5 & $52 *$ & This report \\
\hline F17 & Espagne & c. $568 \mathrm{C}>\mathrm{T}$ & p.Arg190Cys & Exon 5 & 42.5 & {$[4,43]$} \\
\hline F18 & France & c. $568 \mathrm{C}>\mathrm{T}$ & p.Arg190Cys & Exon 5 & NA & {$[4,43]$} \\
\hline F19 & France & c. $568 \mathrm{C}>\mathrm{T}$ & p.Arg190Cys & Exon 5 & NA & {$[4,43]$} \\
\hline F20 & France & c. $575 \mathrm{~A}>\mathrm{G}$ & p.His192Arg & Exon 5 & 44 & This report \\
\hline $\mathrm{F} 21$ & Espagne & c. $632 \mathrm{~A}>\mathrm{G}$ & p.Gln211Arg & Exon 6 & 34.6 & This report \\
\hline $\mathrm{F} 22$ & France & c.666delC & p.Met223fs & Exon 6 & $49 *$ & This report \\
\hline $\mathrm{F} 23$ & Belgium & c. $806 \mathrm{~T}>\mathrm{C}$ & p.Phe269Ser & Exon 7 & 36 & This report \\
\hline $\mathrm{F} 24$ & France & c. $808 \mathrm{G}>\mathrm{T}$ & p.Glu270X & Exon 7 & 56.1 & This report \\
\hline $\mathrm{F} 25$ & France & c. $808 \mathrm{G}>\mathrm{T}$ & p.Glu270X & Exon 7 & NA & This report \\
\hline F26 & Canada & c.810delA & p.Ala271fs & Exon 7 & NA & This report \\
\hline $\mathrm{F} 27$ & France & c. $815 \mathrm{~T}>\mathrm{C}$ & p.Leu272Pro & Exon 7 & 44.4 & This report \\
\hline $\mathrm{F} 28$ & France & c. $821 \mathrm{C}>\mathrm{T}$ & p.Ala274Val & Exon 7 & 45.1 & $\begin{array}{c}\text { Detailed in } \\
\text { [42] }\end{array}$ \\
\hline F29 & Portugal & c. $869 \mathrm{G}>\mathrm{A}$ & p.Cys290Tyr & Exon 7 & 40 & {$[18]$} \\
\hline F30 & France & c. $898 \mathrm{C}>\mathrm{T}$ & p.Arg300X & Exon7 & 66.7 & {$[6]$} \\
\hline F31 & Spain & c. $989 A>G$ & p.Asn330Ser & Exon 8 & 38.7 & [6] \\
\hline F32 & France & c. $1028 \mathrm{~A}>\mathrm{G}$ & p.Gln343Arg & Exon 8 & $47.2 *$ & This report \\
\hline F33 & France & c. $1028 \mathrm{~A}>\mathrm{G}$ & p.Gln343Arg & Exon 8 & NA & This report \\
\hline
\end{tabular}




\begin{tabular}{|c|c|c|c|c|c|c|}
\hline F34 & France & c. $1060 \mathrm{G}>\mathrm{A}$ & p.Gly354Arg & Exon 8 & 46.7 & $\begin{array}{c}{[7,20,24,} \\
38]\end{array}$ \\
\hline F35 & France & c. $1060 \mathrm{G}>\mathrm{A}$ & p.Gly354Arg & Exon 8 & 51.8 & $\begin{array}{c}{[7,20,24,} \\
38]\end{array}$ \\
\hline F36 & France & c. $1060 \mathrm{G}>\mathrm{A}$ & p.Gly354Arg & Exon 8 & NA & $\begin{array}{c}{[7,20,24,} \\
38]\end{array}$ \\
\hline F37 & Tunisia & c. $1108-2 A>G$ & Splice site & Intron 8 & 50.9 & This report \\
\hline F38 & Colombia & c.1121-1123 delTAC & p.Leu374_His375delinsTyr & Exon 9 & NA & This report \\
\hline F39 & Canada & c. $1265 \mathrm{~A}>\mathrm{G}$ & p.Tyr422Cys & Exon 10 & NA & {$[3,14]$} \\
\hline F40 & Israel & c. $1371 \mathrm{G}>\mathrm{A}$ & p.Trp457X & Exon 10 & NA & This report \\
\hline \multicolumn{7}{|c|}{ Sporadic and familial type 2 papillary renal cell cancers } \\
\hline F41 & France & c. $220 \mathrm{G}>\mathrm{C}$ & p.Ala74Pro & Exon 3 & $41.3 *$ & [6] \\
\hline F42 & Mali & c. $426+1 \mathrm{G}>\mathrm{A}$ & Splice site & Intron 4 & $58.2 *$ & This report \\
\hline F43 & Morocco & c. $988 \mathrm{~A}>\mathrm{G}$ & p.Asn330Asp & Exon 8 & $46^{*}$ & This report \\
\hline $\mathrm{F} 44$ & China & c.994delA & p.Thr332fs & Exon 8 & NA & This report \\
\hline
\end{tabular}

Table 1: FH germline mutations identified in the study. (1) FH activity in percentage compared to wild type controls. The activity was determined on lymphoblastoid cell line or (*) on peripheral blood lymphocytes.

\section{Clinical findings}

Clinical data could be recovered for 151 relatives belonging to the 44 families with a $F H$ mutation and are summarized in Table 2. Briefly, cutaneous leiomyomas occurred in 37/44 (84.1\%) FH mutation-positive families that were clinically evaluated by a dermatologist and in 102/151 (67.5\%) gene-carriers. They were multiple in the 65 patients for which detailed data were available, with the exception of a 76 years-old woman who had only a single leiomyoma without any other clinical manifestation. In 8 patients without familial history of MCUL/HLRCC, cutaneous leiomyomas was the only one clinical manifestation. Uterine leiomyomas occurred in 32/44 (72.7\%) FH mutation-positive families and in 76/93 $(81.7 \%)$ affected women and usually were of early onset. In most cases, uterine leiomyomas were multiple, symptomatic and led to hysterectomy before age of 40 years but medical records were obtained only for a few patients. 
Renal tumors occurred in 15/44 (34\%) of families with $F H$ mutation and in $27 / 151$ (17.9\%) of affected members. There were 19 men and 8 women affected with RCC and the age at diagnosis of RCC, known for 21 patients, was an average of 43 years (range: 28-70). In all these patients, RCC was revealed by clinical symptoms (mainly hematuria, abdominal, lumbar or bony pains) and to date no renal tumor was detected in other gene-carriers. Pathological analysis of RCC demonstrated 16 PRCCII (including one sarcomatoid), 2 collecting duct RCC and one sarcomatoid clear-cell RCC. Data were missing for the 8 last patients. Twenty patients (74.1\%) died because of metastatic RCC and the mean age, known for 14 patients, was 44 years (range: 17-66). In four families there was no other clinical manifestation including two families with 4 cases of RCC each and two index cases presenting as sporadic PRCCII. In addition, one patient (family F37), aged 31 years had bilateral atypical renal cysts on CT-scan but no tumor.

The clinical history of the patient with p.Ala274Val mutation was detailed in a very recent paper because of the unique association of cutaneous leiomyomatosis with cutis verticis gyrata, disseminated collagenoma and Charcot-Marie-Tooth's disease. [40]

There was no clear genotype-phenotype correlation specially regarding the occurrence of renal tumors. On the other hand, we observed an intrafamilial phenotypic heterogeneity as illustrated in figure 3. Moreover, genetic testing was positive in five asymptomatic (20-65 years) relatives in which detailed clinical investigations demonstrated no manifestation (figure 3). 


\begin{tabular}{|c|c|c|c|c|c|c|c|c|}
\hline \multirow[t]{2}{*}{ Family } & \multirow[t]{2}{*}{ Mutation } & \multirow[t]{2}{*}{ Patients } & \multirow[t]{2}{*}{$\begin{array}{l}\text { Cutaneous } \\
\text { leiomyomas }\end{array}$} & \multirow[t]{2}{*}{$\begin{array}{c}\text { Uterine } \\
\text { leiomyomas* }\end{array}$} & \multicolumn{4}{|c|}{$\begin{array}{l}\text { Renal Cell Carcinoma } \\
\text { (RCC) }\end{array}$} \\
\hline & & & & & Number & Sex & Age & RCC type \\
\hline \multicolumn{9}{|c|}{ Families with demonstrative manifestations of HLRCC } \\
\hline $\mathrm{F} 1$ & c.1-?_1404+?del & 5 & 5 & $1 / 2$ & 0 & & & \\
\hline $\mathrm{F} 2$ & c.127_128delGA & 1 & 1 & $0 / 0$ & 0 & & & \\
\hline $\mathrm{F} 3$ & $\begin{array}{c}\text { c. } 138+1 \_138+10 \mathrm{~d} \\
\text { el10 }\end{array}$ & 2 & 2 & $2 / 2$ & 0 & & & \\
\hline $\mathrm{F} 4$ & c.147delT & 1 & 1 & $0 / 0$ & 0 & & & \\
\hline F5 & c. $172 \mathrm{C}>\mathrm{T}$ & 4 & 3 & $2 / 4$ & 0 & & & \\
\hline F6 & c. $220 \mathrm{G}>\mathrm{C}$ & 2 & 2 & $1 / 1$ & 0 & & & \\
\hline F7 & c. $220 \mathrm{G}>\mathrm{C}$ & 1 & 1 & NA & 0 & & & \\
\hline F8 & c. $220 \mathrm{G}>\mathrm{C}$ & 1 & 1 & $0 / 1$ & 0 & & & \\
\hline F9 & $\begin{array}{c}\text { c. } 247 \_249+1 \\
\text { delGAGGinsA }\end{array}$ & 1 & 1 & $1 / 1$ & 1 & $\mathrm{~F}$ & 43 & PRCCII \\
\hline F10 & $\begin{array}{c}\text { c. } 247 \_249+1 \\
\text { delGAGGinsA }\end{array}$ & 9 & 6 & $3 / 4$ & 2 & $\begin{array}{l}M \\
M\end{array}$ & $\begin{array}{l}35 \\
52\end{array}$ & $\begin{array}{l}\text { CD RCC } \\
\text { PRCCII }\end{array}$ \\
\hline F11 & c. $250-2 A>G$ & 3 & 2 & $1 / 1$ & 1 & M & 34 & PRCCII \\
\hline F12 & c. $250-2 \mathrm{~A}>\mathrm{G}$ & 1 & 1 & $1 / 1$ & 0 & & & \\
\hline F13 & c. $250-2 A>G$ & 2 & 1 & $2 / 2$ & 0 & & & \\
\hline F14 & c. $298 \mathrm{del} A$ & 2 & 1 & $2 / 2$ & 0 & & & \\
\hline F15 & c. $410 A>G$ & 3 & 2 & $2 / 2$ & 2 & $\begin{array}{l}\mathrm{M} \\
\mathrm{F}\end{array}$ & $\begin{array}{l}65 \\
30\end{array}$ & $\begin{array}{c}\text { NA } \\
\text { SR- } \\
\text { PRCCII }\end{array}$ \\
\hline F16 & c. $503 \mathrm{~T}>\mathrm{C}$ & 4 & 4 & $2 / 2$ & 0 & & & \\
\hline F17 & c. $568 \mathrm{C}>\mathrm{T}$ & 7 & 3 & $7 / 7$ & 0 & & & \\
\hline F18 & c. $568 \mathrm{C}>\mathrm{T}$ & 5 & 4 & $1 / 2$ & 1 & M & NA & NA \\
\hline F19 & c. $568 \mathrm{C}>\mathrm{T}$ & 2 & 1 & $2 / 2$ & 0 & & & \\
\hline $\mathrm{F} 20$ & c. $575 \mathrm{~A}>\mathrm{G}$ & 3 & 3 & $2 / 2$ & 0 & & & \\
\hline $\mathrm{F} 21$ & c. $632 \mathrm{~A}>\mathrm{G}$ & 2 & 1 & $2 / 2$ & 0 & & & \\
\hline $\mathrm{F} 22$ & c.666delC & 4 & 1 & $3 / 4$ & 0 & & & \\
\hline $\mathrm{F} 23$ & c. $806 \mathrm{~T}>\mathrm{C}$ & 1 & 1 & $1 / 1$ & 0 & & & \\
\hline $\mathrm{F} 24$ & c. $808 \mathrm{G}>\mathrm{T}$ & 3 & 2 & $0 / 1$ & 0 & & & \\
\hline $\mathrm{F} 25$ & c. $808 \mathrm{G}>\mathrm{T}$ & 1 & 1 & $0 / 1$ & 0 & & & \\
\hline F26 & c.810delA & 3 & 2 & $2 / 2$ & 1 & M & 49 & NA \\
\hline
\end{tabular}




\begin{tabular}{|c|c|c|c|c|c|c|c|c|}
\hline F27 & c. $815 \mathrm{~T}>\mathrm{C}$ & 4 & 3 & $3 / 3$ & 0 & & & \\
\hline F28 & c. $821 \mathrm{C}>\mathrm{T}$ & 6 & 4 & $2 / 3$ & 0 & & & \\
\hline F29 & c. $869 \mathrm{G}>\mathrm{A}$ & 4 & 1 & $4 / 4$ & 0 & & & \\
\hline F30 & c. $898 \mathrm{C}>\mathrm{T}$ & 6 & 3 & $6 / 6$ & 0 & & & \\
\hline F31 & c. $989 A>G$ & 4 & 3 & $2 / 2$ & 0 & & & \\
\hline F32 & c. $1028 \mathrm{~A}>\mathrm{G}$ & 2 & 2 & $1 / 1$ & 0 & & & \\
\hline F33 & c. $1028 \mathrm{~A}>\mathrm{G}$ & 6 & 5 & $3 / 3$ & 1 & $\mathrm{~F}$ & 34 & PRCCII \\
\hline F34 & c. $1060 \mathrm{G}>\mathrm{A}$ & 7 & 5 & $3 / 4$ & 3 & $\begin{array}{l}\mathrm{M} \\
\mathrm{M} \\
\mathrm{M}\end{array}$ & $\begin{array}{r}59 \\
57 \\
\text { NA }\end{array}$ & $\begin{array}{c}\text { PRCCII } \\
\text { PRCCII } \\
\text { NA }\end{array}$ \\
\hline F35 & c. $1060 \mathrm{G}>\mathrm{A}$ & 4 & 3 & $3 / 4$ & 2 & $\begin{array}{l}\mathrm{F} \\
\mathrm{F}\end{array}$ & $\begin{array}{l}50 \\
52\end{array}$ & $\begin{array}{l}\text { SR RCC } \\
\text { PRCCII }\end{array}$ \\
\hline F36 & c. $1060 \mathrm{G}>\mathrm{A}$ & 5 & 5 & $0 / 2$ & 0 & & & \\
\hline F37 & c. $1108-2 A>G$ & 1 & 1 & $0 / 0$ & 0 & & & \\
\hline F38 & $\begin{array}{c}\text { c.1121-1123 } \\
\text { delAC }\end{array}$ & 5 & NA & $3 / 4$ & 2 & $\begin{array}{l}\mathrm{M} \\
\mathrm{F}\end{array}$ & $\begin{array}{l}40 \\
28\end{array}$ & $\begin{array}{c}\text { NA } \\
\text { PRCCII }\end{array}$ \\
\hline F39 & c. $1265 \mathrm{~A}>\mathrm{G}$ & 1 & 1 & $1 / 1$ & 0 & & & \\
\hline $\mathrm{F} 40$ & c. $1371 \mathrm{G}>\mathrm{A}$ & 17 & 16 & $5 / 5$ & 1 & M & 36 & CD RCC \\
\hline \multicolumn{9}{|c|}{ Sporadic or familial type 2 papillary renal cell cancers } \\
\hline F41 & c. $220 \mathrm{G}>\mathrm{C}$ & 1 & 0 & 0 & 1 & M & 34 & $\begin{array}{c}\mathrm{Sp} \\
\text { PRCCII }\end{array}$ \\
\hline $\mathrm{F} 42$ & c. $426+1 \mathrm{G}>\mathrm{A}$ & 1 & 0 & 0 & 1 & M & 37 & $\begin{array}{c}\text { Sp } \\
\text { PRCCII }\end{array}$ \\
\hline F43 & c. $988 \mathrm{~A}>\mathrm{G}$ & 4 & 0 & 0 & 4 & $\begin{array}{c}\mathrm{M} \\
\mathrm{M} \\
\mathrm{F} \\
\mathrm{M}\end{array}$ & $\begin{array}{l}31 \\
40 \\
59 \\
70\end{array}$ & $\begin{array}{l}\text { PRCCII } \\
\text { PRCCII } \\
\text { PRCCII } \\
\text { PRCCII }\end{array}$ \\
\hline F44 & c.994delA & 4 & 0 & $0 / 1$ & 4 & $\begin{array}{l}\mathrm{M} \\
\mathrm{M} \\
\mathrm{M} \\
\mathrm{F}\end{array}$ & $\begin{array}{l}17 \\
34 \\
45 \\
69\end{array}$ & $\begin{array}{l}\text { PRCCII } \\
\text { PRCCII } \\
\text { PRCCII } \\
\text { PRCCII }\end{array}$ \\
\hline
\end{tabular}

Table 2: Clinical manifestations observed in patients with identified $F H$ mutation. * in women, M: male, F: female, NA: data not available, PRCCII: type 2 papillary renal cell cancers, SR: sarcomatoid, CD: collecting duct, Sp: sporadic. 


\section{DISCUSSION}

The present study reports the identification and analysis of $F H$ mutations, including 21novel, in 40 families with MCUL/HLRCC and, for the first time, in 4 patients with isolated PRCCII. The mutations identified in our series include, at the protein level, 23 (52.3\%) missense mutations, seven (15.9\%) frameshifts, five (11.4\%) nonsense mutations, one (2.3\%) micro deletion/insertion, and eight (18.2\%) sequence variations affecting splice sites. These results are comparable to the $F H$ mutation database; missense mutations are the most common type of germline $F H$ mutation in MCUL/HLRCC families (64\%), followed by frameshifts (14.6\%) and nonsense (12.5\%) mutations. [21] Additionally, we have doubled the number of known splice site mutations (identified in 8 families $(18.2 \%)$ in the present study versus seven (4.9\%) previously described worldwide), emphasizing the importance of careful analysis of non-coding sequences surrounding splice sites. We found only one family with large deletion thus confirming that germline copy loss of $F H$ is a rare genetic event (only four large deletions and one deletion of exon 1 in 144 HLRCC families described). [6]

In order to evaluate the potential deleterious effect of novel nucleotide variations identified in the $F H$ gene, we measured the enzymatic activity of endogenous fumarate hydratase in lymphoblastoid cells derived from patients. Reduction of, at least, 50\% of the enzymatic activity was observed for all mutations tested thus supporting a model of loss of function and haploinsufficiency. Mutant FH proteins have been postulated to exert a dominant negative effect based on FH enzymatic activity [4, 6, 7]; this hypothesis is supported by in vitro over-expression experiments in which the R190H FH mutant induced $60 \%$ inhibition of the endogenous enzymatic activity.[44] As the active FH enzyme is a 
homotetramer, it is theoretically possible that missense mutations may affect $\mathrm{FH}$ activity more drastically than nonsense mutations; these mutations could disrupt the formation of nearly all wild-type homotetramers, leaving only one in $16(1 / 24)$ tetramers composed of exclusively wild-type subunits. Therefore, a missense variant behaving as a strong dominant negative could dramatically reduce enzymatic activity of heterotetrameric FH. Such molecular behavior has never been described, and we did not notice a major difference in the enzymatic activities of missense mutations versus total loss-of-function mutations. Therefore, we conclude that the missense allele products do not exert a strong dominant negative effect in vivo.

Genotype-phenotype relationships between the $F H$ mutational spectrum and HLRCC manifestations have been previously proposed. For example, p.Arg58X FH mutations have been associated with a high frequency of kidney cancers [4], but the carrier of this mutation in our series did not develop RCC by the age of 62 years. The p.Gly354Arg mutation has been reported to predispose patients exclusively to fibroids [7, 45], but we found this mutation in families that displayed various phenotypes of skin lesions and renal cancers. In addition, several mutations that have been described in families with RCCs (p.Arg190Cys, p.Arg300X, p.Asn330Ser) or without RCCs (p.His137Arg) were associated with the opposite phenotype in our study. Phenotype heterogeneity is also observed within families (Figure 3). Therefore, there is to date no convincing evidence of a relationship between the type of $\mathrm{FH}$ mutation and the resulting disease phenotype.

We observed $\mathrm{FH}$ mutations in only $71.4 \%$ (40/56) of the MCUL/HLRCC families included in our study, a lower ratio compared with previous reports. This difference may be due to the recruitment of probands who not fulfill all clinical criteria of the HLRCC disease 
(specially patients with isolated cutaneous leiomyomas or patients with uterine leiomyomas and RCC). On the other hand, these broad inclusion criteria allowed us to identify seven new families with HLRCC that would have not been detected otherwise. Indeed, the recruitment of patients by urologists allowed us to revise the percentage of families with RCC to $40 \%$ from the previously reported $20 \%$ (29/144 families). Concerning the percentage of all individuals with an $F H$ mutation, we observed an RCC incidence of $17.9 \%$, a figure similar to the $10-16 \%$ incidence previously described.[3]

In addition, we describe $F H$ germline mutations in two families with history of PRCCII only (no documented history of leiomyomatosis) and, for the fist time, in two patients with apparent sporadic PRCCII. The p.Ala74Pro mutation (family F41) has been previously reported in the context of HLRCC without RCC [6]; however the splice site mutation c.426+1G>A (family F42), the p.Thr332fs mutation (family F44), and the p.Asn330Asp mutation (family F43) are novel. Interestingly, the unaffected father of family F44 proband's can be considered an obligate carrier, knowing that his mother and two of his brothers developed PRCCII. Consequently, we expanded the $F H$ genotyping to relatives of the patients diagnosed with an $F H$ mutation (58 more family members). As a result, we identified 5 patients with $F H$ germline mutations who lacked any manifestation of the disease similarly to families described in [4].

This question of incomplete penetrance is also raised for the parents of child carriers of homozygous/compound heterozygous FH germline mutations, who are obligate heterozygous $\mathrm{FH}$ mutation carriers. These observations suggest low penetrance but need confirmation by analyzing a larger family set. As accumulation of fumarate subsequent to FH activity loss may contribute to the pathology [33], incomplete penetrance could thus be 
due to individual variation in $\mathrm{FH}$ enzymatic activity in relation with other host factors. Therefore, we compared the FH enzymatic activity of cells from patients of the same family exhibiting severe phenotypes or no to very mild phenotypes. We obtained comparable results with the same loss of $\mathrm{FH}$ activity in the asymptomatic patients (Supplementary Table 2).

Taken together, these results demonstrate that the phenotypic spectrum of $F H$ carriers is broader than expected, extending from asymptomatic to severe disease with multiple tumors including RCC. Additional genetic or environmental modifying factors may play an important role in the development of the disease. Fine mapping and haplotype analysis surrounding the $F H$ gene failed to identify a genetic modifier for RCC risk in HLRCC families (probands of families F11 and F34) [24], and a differential transcriptional study of asymptomatic versus symptomatic patients showed no significant differences (families F10, F11, and F41, data not shown). It could be interesting to perform a metabolomic study in patients with low clinical affection to investigate whether they have developed alternative pathways to compensate for the $F H$ mutation. Hence, due to the incomplete penetrance of HLRCC, genetic testing of $F H$ should be conducted more widely, and should be applied to patients with apparent sporadic PRCCII when the patient is less than 40 years old at diagnosis or when the histology is characteristics of HLRCC as recently defined by Merino et al. (large nucleus with proeminent oriangiophilic or eosinophilic nucleolus surrounded by a clear halo). [25] This expanded testing regimen could allow the discovery of previously undiagnosed HLRCC families, leading to appropriate clinical management including dermatological surveillance and gynecological examination in women at potential risk for early hysterectomy. Indeed, careful skin examination of the proband of family F41 
(affected with an apparent sporadic PRCCII) was performed after $F H$ mutation identification and revealed a small cutaneous leiomyoma.

In addition to the potential implications for presymptomatic diagnosis, identification of patients with $F H$ germline mutations could also be critical for the determination of the appropriate treatment of advanced PRCCII. Indeed, as hereditary PRCCIIs are characterized by HIF overexpression and activation of angiogenesis [32], it would be useful to explore the efficacy of anti-angiogenic drugs in patients affected by such tumors. Recently, prolonged progression-free survival times were reported in 2/12 patients with apparent sporadic PRCCII treated by sunitinib, a novel tyrosine kinase inhibitor. [46] Further investigation is needed, but preliminary observation showed that one of these two patients presented cutaneous leiomyomas and is certainly a carrier of a germline $\mathrm{FH}$ mutation. Thus, it would be of great interest to explore the potential predictive role of $\mathrm{FH}$ mutations in therapy response.

\section{ACKNOWLEDGMENTS}

We would like to thank all participating patients and their family members. We also thank Prof. J.P. Grünfeld for his constant support. We also acknowledge the contribution of the IGR Biobank for providing their samples.

\section{COMPETING INTEREST}

None. 


\section{FUNDING}

This work was supported by grants from the French National Cancer Institute ("INCa", PNES Rein; and Réseau National Prédispositions héréditaires au cancer du rein), the French Ligue Nationale contre le Cancer (Comités du Cher, de l'Indre et de l'Allier) and the Association de Recherches contre le Cancer (ARC).

\section{LICENCE STATEMENT}

"The Corresponding Author has the right to grant on behalf of all authors and does grant on behalf of all authors, an exclusive licence (or non exclusive for government employees) on a worldwide basis to the BMJ Publishing Group Ltd to permit this article (if accepted) to be published in JMG and any other BMJPGL products and sublicences such use and exploit all subsidiary rights, as set out in our licence (http://group.bmj.com/products/journals/instructions-for-authors/licence-forms)." 


\section{REFERENCES}

1. Lehtonen HJ, Kiuru M, Ylisaukko-Oja SK, Salovaara R, Herva R, Koivisto PA, Vierimaa O, Aittomaki K, Pukkala E, Launonen V, Aaltonen LA. Increased risk of cancer in patients with fumarate hydratase germline mutation. J Med Genet 2006;43:523-6.

2. Launonen V, Vierimaa O, Kiuru M, Isola J, Roth S, Pukkala E, Sistonen P, Herva $\mathrm{R}$, Aaltonen LA. Inherited susceptibility to uterine leiomyomas and renal cell cancer. Proc Natl Acad Sci U S A 2001;98:3387-92.

3. Toro JR, Nickerson ML, Wei MH, Warren MB, Glenn GM, Turner ML, Stewart L, Duray P, Tourre O, Sharma N, Choyke P, Stratton P, Merino M, Walther MM, Linehan WM, Schmidt LS, Zbar B. Mutations in the fumarate hydratase gene cause hereditary leiomyomatosis and renal cell cancer in families in North America. Am J Hum Genet 2003;73:95-106.

4. Wei MH, Toure O, Glenn GM, Pithukpakorn M, Neckers L, Stolle C, Choyke P, Grubb R, Middelton L, Turner ML, Walther MM, Merino MJ, Zbar B, Linehan WM, Toro JR. Novel mutations in FH and expansion of the spectrum of phenotypes expressed in families with hereditary leiomyomatosis and renal cell cancer. $J$ Med Genet 2006;43:18-27.

5. Alam NA, Olpin S, Leigh IM. Fumarate hydratase mutations and predisposition to cutaneous leiomyomas, uterine leiomyomas and renal cancer. Br J Dermatol 2005;153:11-7.

6. Tomlinson IP, Alam NA, Rowan AJ, Barclay E, Jaeger EE, Kelsell D, Leigh I, Gorman P, Lamlum H, Rahman S, Roylance RR, Olpin S, Bevan S, Barker K, Hearle N, Houlston RS, Kiuru M, Lehtonen R, Karhu A, Vilkki S, Laiho P, Eklund C, Vierimaa O, Aittomaki K, Hietala M, Sistonen P, Paetau A, Salovaara R, Herva $\mathrm{R}$, Launonen V, Aaltonen LA. Germline mutations in FH predispose to dominantly inherited uterine fibroids, skin leiomyomata and papillary renal cell cancer. Nat Genet 2002;30:406-10.

7. Alam NA, Rowan AJ, Wortham NC, Pollard PJ, Mitchell M, Tyrer JP, Barclay E, Calonje E, Manek S, Adams SJ, Bowers PW, Burrows NP, Charles-Holmes R, Cook LJ, Daly BM, Ford GP, Fuller LC, Hadfield-Jones SE, Hardwick N, Highet AS, Keefe M, MacDonald-Hull SP, Potts ED, Crone M, Wilkinson S, CamachoMartinez F, Jablonska S, Ratnavel R, MacDonald A, Mann RJ, Grice K, Guillet G, Lewis-Jones MS, McGrath H, Seukeran DC, Morrison PJ, Fleming S, Rahman S, Kelsell D, Leigh I, Olpin S, Tomlinson IP. Genetic and functional analyses of FH mutations in multiple cutaneous and uterine leiomyomatosis, hereditary leiomyomatosis and renal cancer, and fumarate hydratase deficiency. Hum Mol Genet 2003;12:1241-52.

8. Martinez-Mir A, Glaser B, Chuang GS, Horev L, Waldman A, Engler DE, Gordon D, Spelman LJ, Hatzibougias I, Green J, Christiano AM, Zlotogorski A. Germline fumarate hydratase mutations in families with multiple cutaneous and uterine leiomyomata. J Invest Dermatol 2003;121:741-4. 
9. Alam NA, Olpin S, Rowan A, Kelsell D, Leigh IM, Tomlinson IP, Weaver T. Missense mutations in fumarate hydratase in multiple cutaneous and uterine leiomyomatosis and renal cell cancer. J Mol Diagn 2005;7:437-43.

10. Chan I, Wong T, Martinez-Mir A, Christiano AM, McGrath JA. Familial multiple cutaneous and uterine leiomyomas associated with papillary renal cell cancer. Clin Exp Dermatol 2005;30:75-8.

11. Matyakhina L, Freedman RJ, Bourdeau I, Wei MH, Stergiopoulos SG, Chidakel A, Walther M, Abu-Asab M, Tsokos M, Keil M, Toro J, Linehan WM, Stratakis CA. Hereditary leiomyomatosis associated with bilateral, massive, macronodular adrenocortical disease and atypical cushing syndrome: a clinical and molecular genetic investigation. J Clin Endocrinol Metab 2005;90:3773-9.

12. Badeloe S, van Geel M, van Steensel MA, Bastida J, Ferrando J, Steijlen PM, Frank J, Poblete-Gutierrez P. Diffuse and segmental variants of cutaneous leiomyomatosis: novel mutations in the fumarate hydratase gene and review of the literature. Exp Dermatol 2006;15:735-41.

13. Chuang GS, Martinez-Mir A, Engler DE, Gmyrek RF, Zlotogorski A, Christiano AM. Multiple cutaneous and uterine leiomyomata resulting from missense mutations in the fumarate hydratase gene. Clin Exp Dermatol 2006;31:118-21.

14. Pithukpakorn M, Wei MH, Toure O, Steinbach PJ, Glenn GM, Zbar B, Linehan WM, Toro JR. Fumarate hydratase enzyme activity in lymphoblastoid cells and fibroblasts of individuals in families with hereditary leiomyomatosis and renal cell cancer. J Med Genet 2006;43:755-62.

15. Ylisaukko-oja SK, Kiuru M, Lehtonen HJ, Lehtonen R, Pukkala E, Arola J, Launonen V, Aaltonen LA. Analysis of fumarate hydratase mutations in a population-based series of early onset uterine leiomyosarcoma patients. Int J Cancer 2006;119:283-7.

16. Campione E, Terrinoni A, Orlandi A, Codispoti A, Melino G, Bianchi L, Mazzotta A, Garaci FG, Ludovici A, Chimenti S. Cerebral cavernomas in a family with multiple cutaneous and uterine leiomyomas associated with a new mutation in the fumarate hydratase gene. J Invest Dermatol 2007;127:2271-3.

17. Lehtonen HJ, Blanco I, Piulats JM, Herva R, Launonen V, Aaltonen LA. Conventional renal cancer in a patient with fumarate hydratase mutation. Hum Pathol 2007;38:793-6.

18. Makino T, Nagasaki A, Furuichi M, Matsui K, Watanabe H, Sawamura D, Shimizu $\mathrm{H}$, Shimizu T. Novel mutation in a fumalate hydratase gene of a Japanese patient with multiple cutaneous and uterine leiomyomatosis. J Dermatol Sci 2007;48:151-3.

19. Ahvenainen T, Lehtonen HJ, Lehtonen R, Vahteristo P, Aittomaki K, Baynam G, Dommering C, Eng C, Gruber SB, Gronberg H, Harvima R, Herva R, Hietala M, Kujala M, Kaariainen H, Sunde L, Vierimaa O, Pollard PJ, Tomlinson IP, Bjorck E, Aaltonen LA, Launonen V. Mutation screening of fumarate hydratase by multiplex ligation-dependent probe amplification: detection of exonic deletion in a patient with leiomyomatosis and renal cell cancer. Cancer Genet Cytogenet 2008;183:83-8. 
20. Badeloe S, Bladergroen RS, Jonkman MF, Burrows NP, Steijlen PM, PobleteGutierrez P, van Steensel MA, van Geel M, Frank J. Hereditary multiple cutaneous leiomyoma resulting from novel mutations in the fumarate hydratase gene. $J$ Dermatol Sci 2008;51:139-43.

21. Bayley JP, Launonen V, Tomlinson IP. The FH mutation database: an online database of fumarate hydratase mutations involved in the MCUL (HLRCC) tumor syndrome and congenital fumarase deficiency. BMC Med Genet 2008;9:20.

22. Huter E, Wortham NC, Hartschuh W, Enk A, Jappe U. Single base mutation in the fumarate hydratase gene leading to segmental cutaneous leiomyomatosis. Acta Derm Venereol 2008;88:63-5.

23. Onder M, Glenn G, Adisen E, Keseroglu O, Sahin S, Ataoglu O, Akkaya B, Toro JR. Cutaneous papules, uterine fibroids, and renal cell cancer: one family's tale. Lancet 2010;375:170.

24. Vahteristo P, Koski TA, Naatsaari L, Kiuru M, Karhu A, Herva R, Sallinen SL, Vierimaa O, Bjorck E, Richard S, Gardie B, Bessis D, Van Glabeke E, Blanco I, Houlston R, Senter L, Hietala M, Aittomaki K, Aaltonen LA, Launonen V, Lehtonen R. No evidence for a genetic modifier for renal cell cancer risk in HLRCC syndrome. Fam Cancer 2010;9:245-51.

25. Merino MJ, Torres-Cabala C, Pinto P, Linehan WM. The morphologic spectrum of kidney tumors in hereditary leiomyomatosis and renal cell carcinoma (HLRCC) syndrome. Am J Surg Pathol 2007;31:1578-85.

26. Yang XJ, Tan MH, Kim HL, Ditlev JA, Betten MW, Png CE, Kort EJ, Futami K, Furge KA, Takahashi M, Kanayama HO, Tan PH, Teh BS, Luan C, Wang K, Pins M, Tretiakova M, Anema J, Kahnoski R, Nicol T, Stadler W, Vogelzang NG, Amato R, Seligson D, Figlin R, Belldegrun A, Rogers CG, Teh BT. A molecular classification of papillary renal cell carcinoma. Cancer Res 2005;65:5628-37.

27. Bourgeron T, Chretien D, Poggi-Bach J, Doonan S, Rabier D, Letouze P, Munnich A, Rotig A, Landrieu P, Rustin P. Mutation of the fumarase gene in two siblings with progressive encephalopathy and fumarase deficiency. J Clin Invest 1994;93:2514-8.

28. Coughlin EM, Christensen E, Kunz PL, Krishnamoorthy KS, Walker V, Dennis NR, Chalmers RA, Elpeleg ON, Whelan D, Pollitt RJ, Ramesh V, Mandell R, Shih VE. Molecular analysis and prenatal diagnosis of human fumarase deficiency. Mol Genet Metab 1998;63:254-62.

29. Gellera C, Uziel G, Rimoldi M, Zeviani M, Laverda A, Carrara F, DiDonato S. Fumarase deficiency is an autosomal recessive encephalopathy affecting both the mitochondrial and the cytosolic enzymes. Neurology 1990;40:495-9.

30. Weaver TM, Levitt DG, Donnelly MI, Stevens PP, Banaszak LJ. The multisubunit active site of fumarase C from Escherichia coli. Nat Struct Biol 1995;2:654-62.

31. Weaver T, Lees M, Zaitsev V, Zaitseva I, Duke E, Lindley P, McSweeny S, Svensson A, Keruchenko J, Keruchenko I, Gladilin K, Banaszak L. Crystal structures of native and recombinant yeast fumarase. J Mol Biol 1998;280:431-42. 
32. Isaacs JS, Jung YJ, Mole DR, Lee S, Torres-Cabala C, Chung YL, Merino M, Trepel J, Zbar B, Toro J, Ratcliffe PJ, Linehan WM, Neckers L. HIF overexpression correlates with biallelic loss of fumarate hydratase in renal cancer: novel role of fumarate in regulation of HIF stability. Cancer Cell 2005;8:143-53.

33. Pollard PJ, Briere JJ, Alam NA, Barwell J, Barclay E, Wortham NC, Hunt T, Mitchell M, Olpin S, Moat SJ, Hargreaves IP, Heales SJ, Chung YL, Griffiths JR, Dalgleish A, McGrath JA, Gleeson MJ, Hodgson SV, Poulsom R, Rustin P, Tomlinson IP. Accumulation of Krebs cycle intermediates and over-expression of HIF1alpha in tumours which result from germline FH and SDH mutations. Hum Mol Genet 2005;14:2231-9.

34. Pollard PJ, Spencer-Dene B, Shukla D, Howarth K, Nye E, El-Bahrawy M, Deheragoda M, Joannou M, McDonald S, Martin A, Igarashi P, Varsani-Brown S, Rosewell I, Poulsom R, Maxwell P, Stamp GW, Tomlinson IP. Targeted inactivation of fh1 causes proliferative renal cyst development and activation of the hypoxia pathway. Cancer Cell 2007;11:311-9.

35. Sudarshan S, Sourbier C, Kong HS, Block K, Valera Romero VA, Yang Y, Galindo C, Mollapour M, Scroggins B, Goode N, Lee MJ, Gourlay CW, Trepel J, Linehan WM, Neckers L. Fumarate hydratase deficiency in renal cancer induces glycolytic addiction and hypoxia-inducible transcription factor 1alpha stabilization by glucosedependent generation of reactive oxygen species. Mol Cell Biol 2009;29:4080-90.

36. Kiuru M, Lehtonen R, Arola J, Salovaara R, Jarvinen H, Aittomaki K, Sjoberg J, Visakorpi T, Knuutila S, Isola J, Delahunt B, Herva R, Launonen V, Karhu A, Aaltonen LA. Few FH mutations in sporadic counterparts of tumor types observed in hereditary leiomyomatosis and renal cell cancer families. Cancer Res 2002;62:4554-7.

37. Parmentier L, Tomlinson I, Happle R, Borradori L. Evidence for a new fumarate hydratase gene mutation in a unilateral type 2 segmental leiomyomatosis.

Dermatology 2010;221:149-53.

38. Smit DL, Mensenkamp AR, Badeloe S, Breuning MH, Simon ME, van Spaendonck KY, Aalfs CM, Post JG, Shanley S, Krapels IP, Hoefsloot LH, van Moorselaar RJ, Starink TM, Bayley JP, Frank J, van Steensel MA, Menko FH. Hereditary leiomyomatosis and renal cell cancer in families referred for fumarate hydratase germline mutation analysis. Clin Genet 2011;79:49-59.

39. Livak KJ, Schmittgen TD. Analysis of relative gene expression data using real-time quantitative PCR and the 2(-Delta Delta C(T)) Method. Methods 2001;25:402-8.

40. Hill RLaB, R.A. . Fumarase: [EC 4.2.1.2 L-Malate hydro-lyase]. Methods Enzymol 1969;13:91-99.

41. Rustin P, Chretien D, Bourgeron T, Gerard B, Rotig A, Saudubray JM, Munnich A. Biochemical and molecular investigations in respiratory chain deficiencies. Clin Chim Acta 1994;228:35-51. 
42. Marque M, Gardie B, Bressac de Paillerets B, Rustin P, Guillot B, Richard S, Bessis D. Novel FH mutation in a patient with cutaneous leiomyomatosis associated with cutis verticis gyrata, eruptive collagenoma and Charcot-Marie-Tooth's disease. $\mathrm{Br} J$ Dermatol 2010;163:1337-9.

43. Smit DL, Mensenkamp AR, Badeloe S, Breuning MH, Simon ME, van Spaendonck KY, Aalfs CM, Post JG, Shanley S, Krapels IP, Hoefsloot LH, van Moorselaar RJ, Starink TM, Bayley JP, Frank J, van Steensel MA, Menko FH. Hereditary leiomyomatosis and renal cell cancer in families referred for fumarate hydratase germline mutation analysis. Clin Genet 2010.

44. Chuang GS, Martinez-Mir A, Geyer A, Engler DE, Glaser B, Cserhalmi-Friedman PB, Gordon D, Horev L, Lukash B, Herman E, Cid MP, Brenner S, Landau M, Sprecher E, Garcia Muret MP, Christiano AM, Zlotogorski A. Germline fumarate hydratase mutations and evidence for a founder mutation underlying multiple cutaneous and uterine leiomyomata. J Am Acad Dermatol 2005;52:410-6.

45. Lorenzato A, Olivero M, Perro M, Briere JJ, Rustin P, Di Renzo MF. A cancerpredisposing "hot spot" mutation of the fumarase gene creates a dominant negative protein. Int J Cancer 2008;122:947-51.

46. Alam NA, Barclay E, Rowan AJ, Tyrer JP, Calonje E, Manek S, Kelsell D, Leigh I, Olpin S, Tomlinson IP. Clinical features of multiple cutaneous and uterine leiomyomatosis: an underdiagnosed tumor syndrome. Arch Dermatol 2005;141:199-206.

47. Choueiri TK, Plantade A, Elson P, Negrier S, Ravaud A, Oudard S, Zhou M, Rini BI, Bukowski RM, Escudier B. Efficacy of sunitinib and sorafenib in metastatic papillary and chromophobe renal cell carcinoma. J Clin Oncol 2008;26:127-31. 


\section{LEGENDS OF FIGURES}

Figure 1. Genomic rearrangement study. A: Amplification plots obtained by real-time PCR for exon 2 of $F H$ and for the control gene (exon 11 of $B R C A 1$ ) in proband of family F1. B: Gene copy number for all $F H$ exons detected in control DNA and in patient's DNA.

Figure 2. Alignment of $\mathrm{FH}$ protein across ten species using the Multalin interface. (http://bioinfo.genopole-toulouse.prd.fr/multalin/). From top to bottom: Homo sapiens, macaque, rat, mouse, dog, chicken, chimpanzee, Caenorhabditis elegans, Saccharomyces cerevisiae, and Escherichia coli. High-consensus residues (90\% conservation) are represented as white font on a red background; low-consensus residues (50\% conservation) are in red font; non-conserved residues are in black font. Missense mutations identified in this study are indicated, and underlines highlight novel mutations

Figure 3. Pedigrees of representative families with HLRCC illustrating phenotypic heterogeneity. Solid symbols represent affected family members, and a slash indicate deceased family members. Probands are identified by an arrow. The result of the genetic testing is indicated: + (mutated) or WT (wild type). 

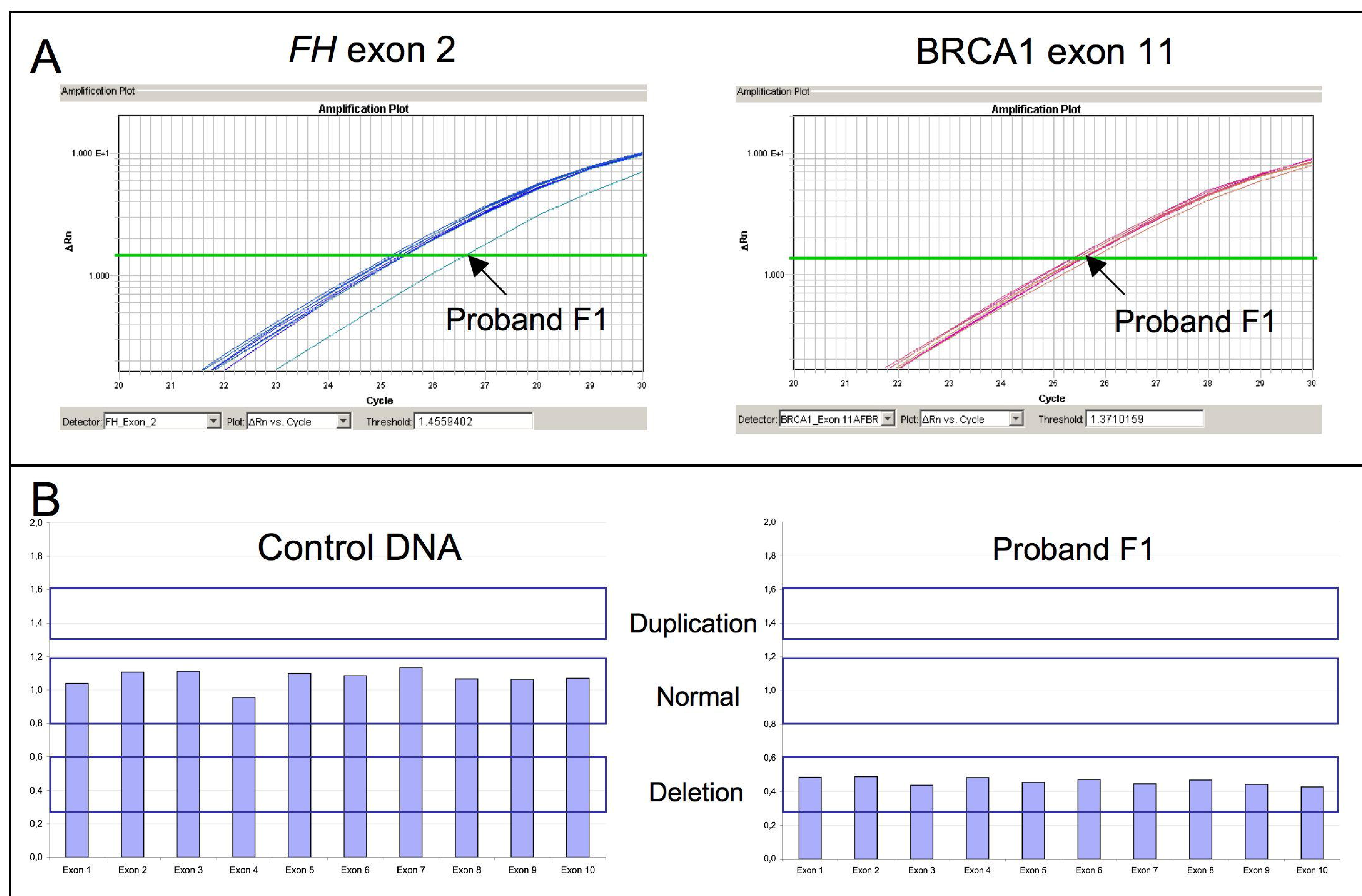

Figure 1 
A74P

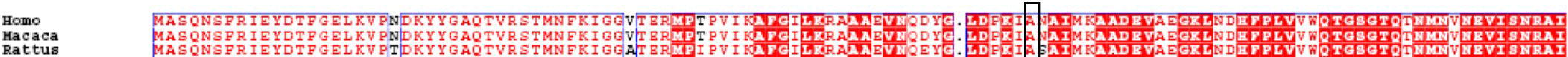

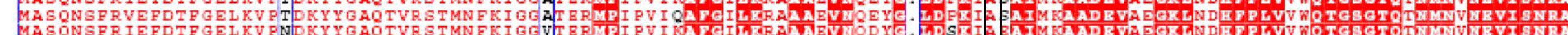

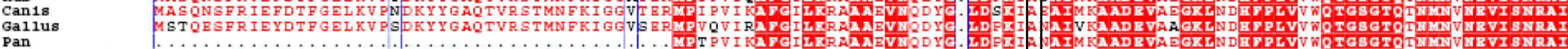

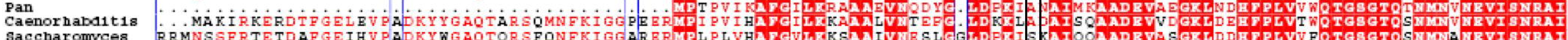
Saccharomyces
consensus

$$
\text { H137R } \quad \underline{L 168 P} \text { R190C } \quad \text { H192R Q211R }
$$

Homo
Hacaca
Rattus

Hus
Can1s
Ga11us

Caenornabd1t18

Saccharomyces
consensus $>50$
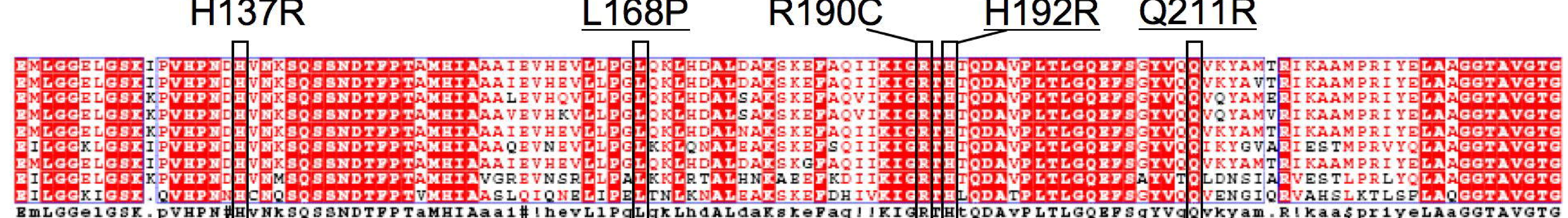

F269S L272P A274V C290Y

N330D N330S Q343R G354R \begin{tabular}{l|l} 
Homo \\
Hacaca \\
Rattus \\
Hus \\
Can1s \\
Ga11us \\
Pan \\
Caenorhaba1t1s \\
Sacharomyces \\
consensus $>50$
\end{tabular}
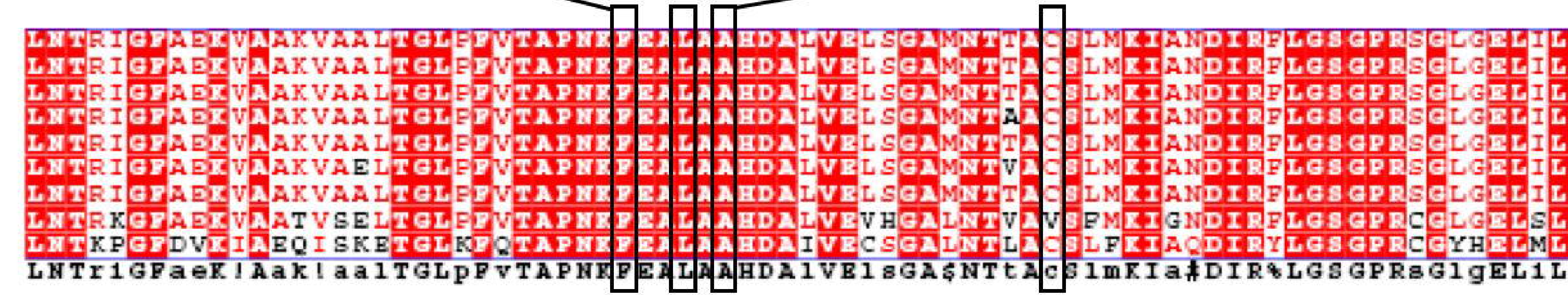

सas

\section{Y422C}

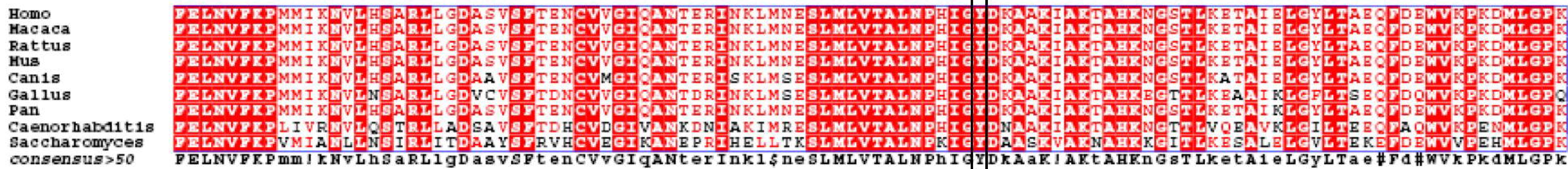




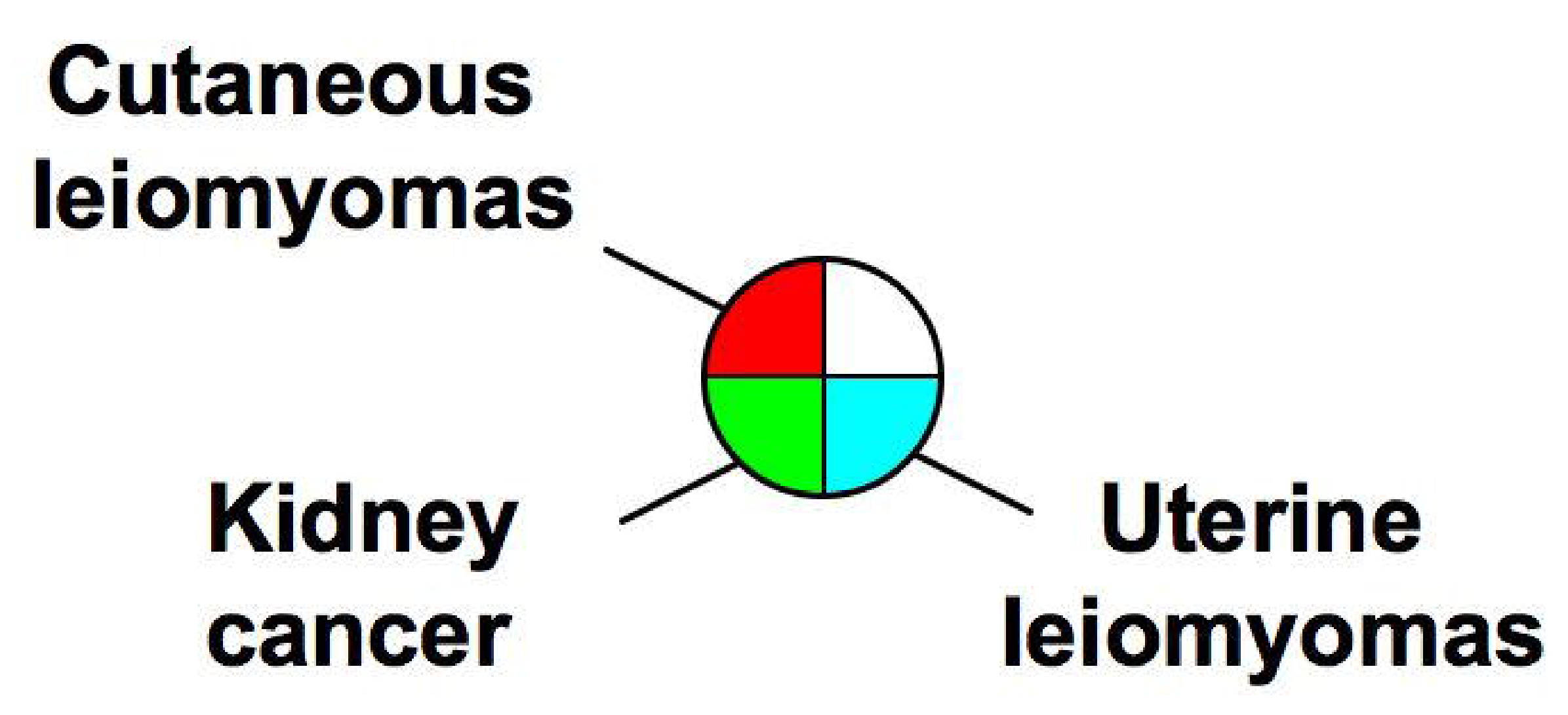

Family 11:

c.250-2A>G

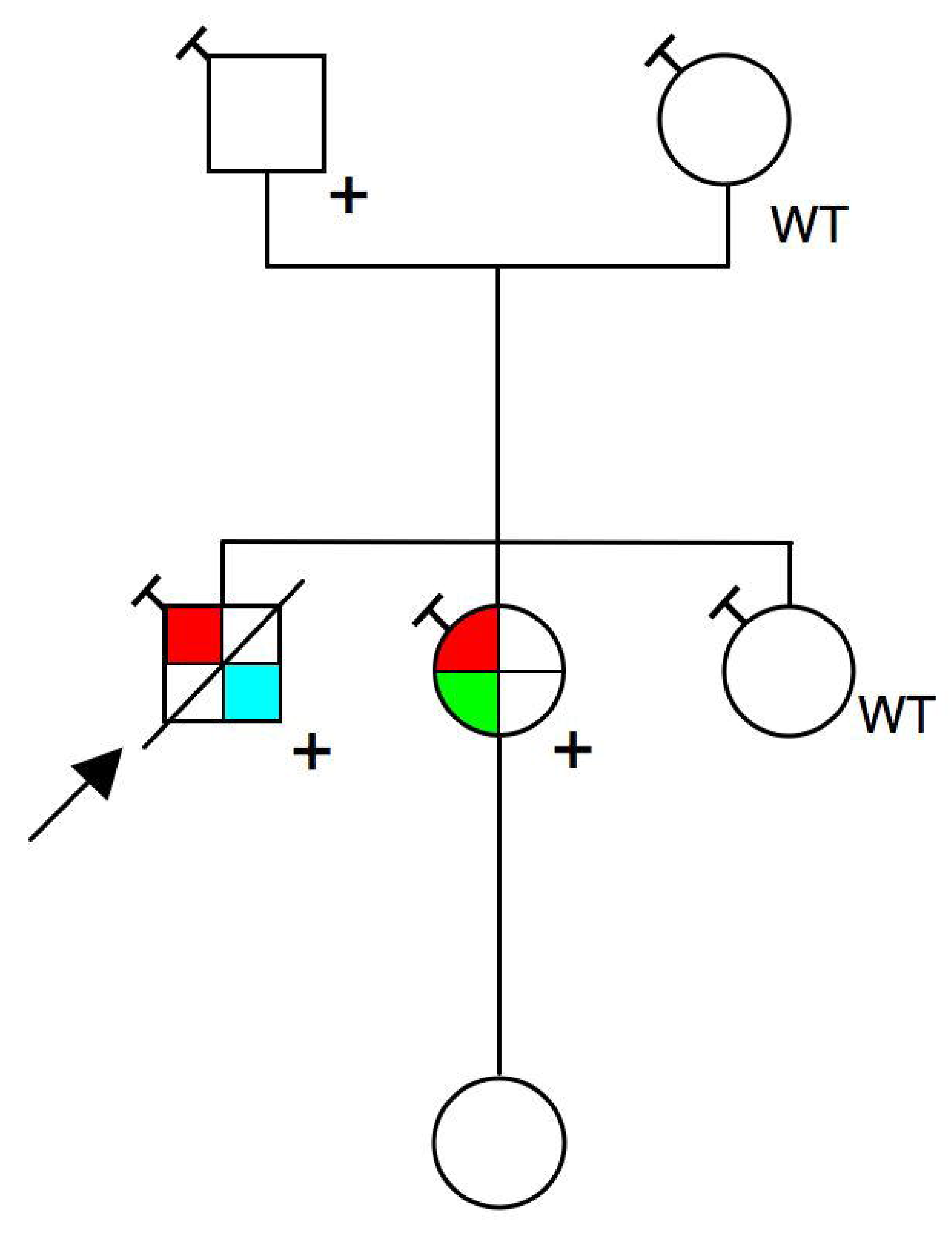

Family 41: c.220G $>$ C, p.Asp74Pro

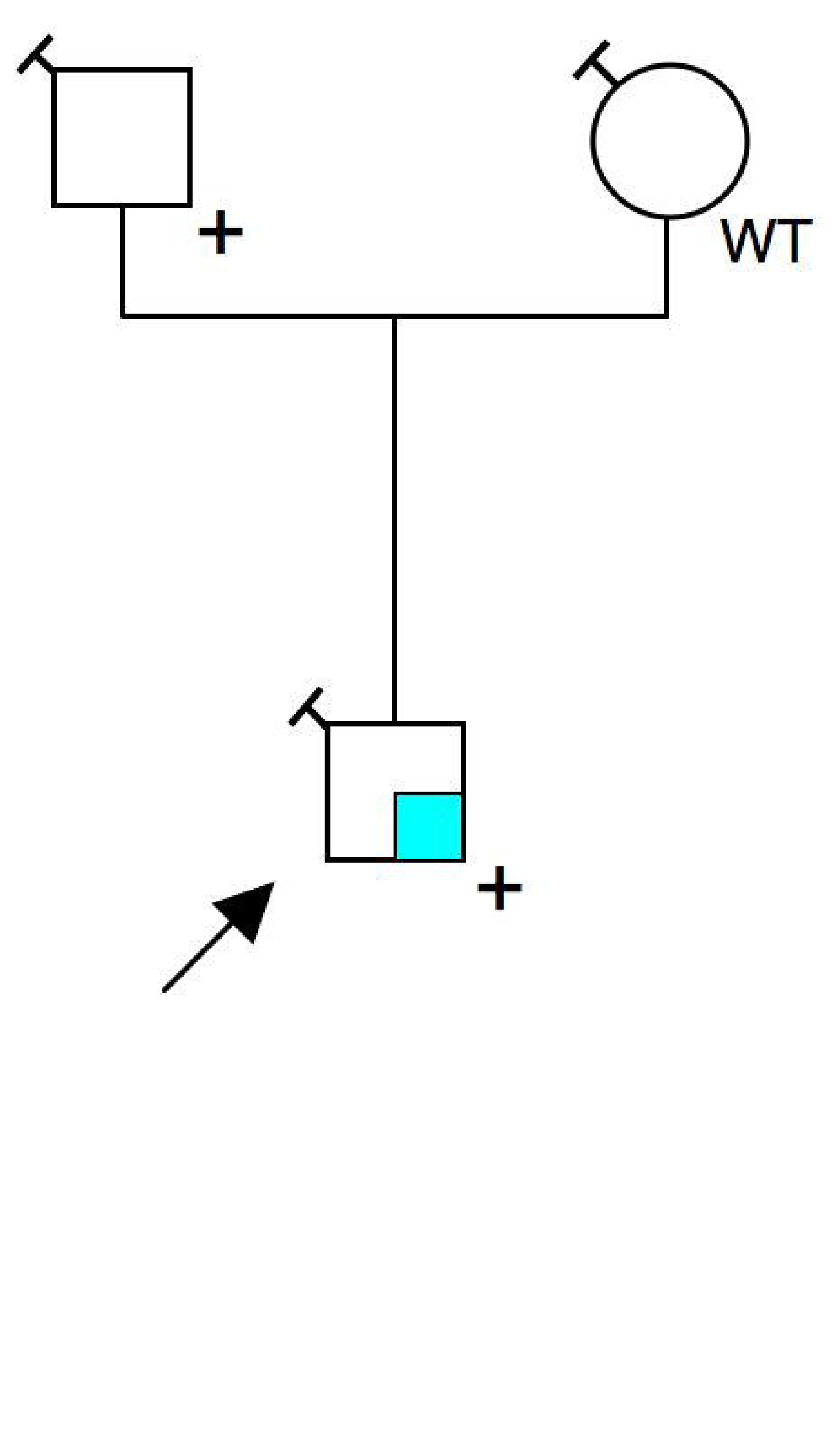

Family 10:

c.247_249+1 delGAGGinsA

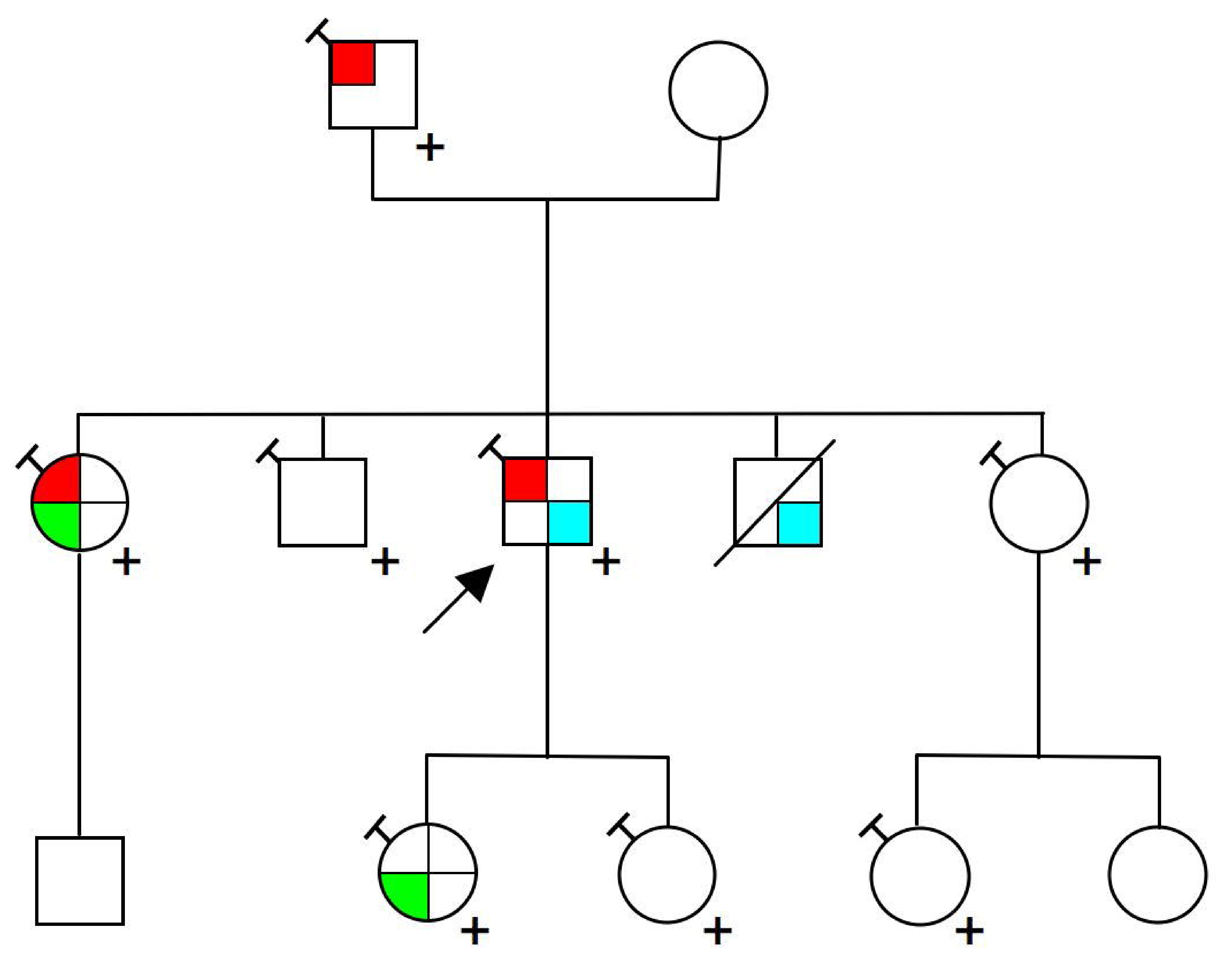

Figure 3 